\title{
Homothetic and Conformal Symmetries of Solutions to Einstein's Equations
}

\author{
D. Eardley, ${ }^{1}$ J. Isenberg, ${ }^{2}$ J. Marsden ${ }^{3}$ and V. Moncrief ${ }^{4}$ \\ 1 Institute for Theoretical Physics, University of California, Santa Barbara, California, 93106, USA \\ 2 Department of Mathematics, University of Oregon, Eugene, Oregon 97403, USA \\ 3 Department of Mathematics, University of California, Berkeley, California 94720, USA \\ 4 Department of Mathematics and Department of Physics, Yale University, P.O. Box 6666, New \\ Haven, Connecticut 06511, USA
}

\begin{abstract}
We present several results about the nonexistence of solutions of Einstein's equations with homothetic or conformal symmetry. We show that the only spatially compact, globally hyperbolic spacetimes admitting a hypersurface of constant mean extrinsic curvature, and also admitting an infinitesimal proper homothetic symmetry, are everywhere locally flat; this assumes that the matter fields either obey certain energy conditions, or are the Yang-Mills or massless Klein-Gordon fields. We find that the only vacuum solutions admitting an infinitesimal proper conformal symmetry are everywhere locally flat spacetimes and certain plane wave solutions. We show that if the dominant energy condition is assumed, then Minkowski spacetime is the only asymptotically flat solution which has an infinitesimal conformal symmetry that is asymptotic to a dilation. In other words, with the exceptions cited, homothetic or conformal Killing fields are in fact Killing in spatially compact or asymptotically flat spactimes. In the conformal procedure for solving the initial value problem, we show that data with infinitesimal conformal symmetry evolves to a spacetime with full isometry.
\end{abstract}

\section{Introduction}

Virtually all explicitly known spacetime solutions of Einstein's equations admit some nontrivial isometry group. This is not surprising since the Einstein equations are very difficult to solve, and isometries simplify them considerably. While much physical insight on astrophysical and cosmological questions has been obtained from the study of spacetimes with lots of symmetry, it clearly would be useful to examine solutions with a smaller isometry group, or even a trivial one. One possible way for reducing spacetime symmetry without giving up all the simplifications it provides is to replace spacetime isometries with spacetime conformal symmetries or homothetic symmetries. A conformal symmetry preserves the metric up to a general point-dependent scale factor, while for a homothetic symmetry the scale factor must be constant. 
There are many solutions known with conformal symmetry, but in most of these the symmetry is actually homothetic, and among the latter no solutions are either spatially compact or asymptotically flat. These results are striking enough to suggest the conjecture that, in most cases, conformal symmetries are homothetic symmetries, and that, in most spatially compact or asymptotically flat cases, conformal or homothetic symmetries are full isometries. In this paper we prove these conjectures, under some technical assumptions.

For spatially compact spacetimes, the main restrictions we make are that spacetime is globally hyperbolic, and that spacetime admits a hypersurface of constant mean extrinsic curvature. The former restriction is reasonable in a study of solutions to Einstein's equations. The latter restriction is less well motivated, although recent work of Gerhardt [1] (see also [2]) indicates that it fails only in pathological cases. Under these restrictions, and under some energy conditions on the stress tensor entering into Einstein's equations, we show in Theorem 1 (Sect. 3) that the only solutions with proper homothetic symmetry are spacetimes with the metric

$$
d s^{2}=e^{\lambda t}\left(-d t^{2}+h_{a b} d x^{a} d x^{b}\right),
$$

where $h_{a b} d x^{a} d x^{b}$ is a 3-dimensional Riemannian metric of constant negative curvature on a compact manifold, and $\lambda$ is a constant; there are various choices for the manifold topology [3]. We will refer to a solution (1.1) as an "expanding hyperbolic spacetime." Such a solution is everywhere locally flat. It is just the quotient of Minkowski spacetime by some discrete subgroup of the Lorentz group, and is therefore essentially trivial. We also present similar results for the Einstein equations coupled to the Yang-Mills equations or the massless Klein-Gordon equations.

The existence of solutions of Einstein's equations with homothetic symmetry is naturally suggested by the fact that the Einstein tensor is itself invariant under homothetic transformations. The nonexistence (almost) of spatially compact solutions is a consequence of the fact that a spatially compact spacetime almost always defines an intrinsic scale, e.g., some measure of the size at maximum expansion, or the maximum proper length of any timelike geodesic. Such an intrinsic scale cannot change under scale transformations, and thus homothetic symmetry is spoiled.

In Sect. 4 we turn to proper conformal symmetries. The Einstein tensor is not invariant under proper conformal transformations, and so these are not natural symmetries of Einstein's equations. Thus solutions with conformal symmetry should be absent or "accidental." Nevertheless, some solutions are known for the Einstein equations with matter that have conformal symmetry. To avoid these counterexamples, we restrict attention to the vacuum Einstein equations. In the completely local Theorem 3 we give all solutions which have proper conformal symmetry - these are either everywhere locally flat, like (1.1) above, or else are of a particular algebraically special form [4],

$$
d s^{2}=-2 H\left(u, x^{C}\right) d u^{2}-2 d u d r+\delta_{A B} d x^{A} d x^{B},
$$

where $A, B, C, \ldots=2,3$, and the vacuum Einstein equations require that $H$ satisfy 
the 2-dimensional Laplace equation in the $\left\{x^{C}\right\}$,

$$
\delta^{A B} \partial_{A} \partial_{B} H\left(u, x^{C}\right)=0 ;
$$

the further conditions which ensure a conformal symmetry are described in Appendix A. We shall refer to solutions (1.2) as "plane-fronted waves."

The asymptotically flat case is discussed in Sect. 5. We show that Minkowski spacetime itself is the only asymptotically flat solution admitting proper conformal or homothetic symmetries, which are asymptotic to the scale transformation (or dilation) symmetry of flat spacetime. The conformal case reduces to the homothetic case as in Sect. 4. In turn, a proper homothetic symmetry would be spoiled by the existence of a nonzero ADM mass, which is an intrinsically defined scale of spacetime. But zero ADM mass implies that spacetime is flat by the positive energy theorem.

Part of the motivation for this work came originally from a question about "linearization stability" of solutions of Einstein's equations. As is well known from the work of Fischer, Marsden, Moncrief and Arms [5-7], the only spatially compact solutions of Einstein's equations which are not linearization stable are those containing isometries. Yet, in their work on the conformal procedure for solving the constraint equations, York and O'Murchadha [8] found linearization instability at data which seem to have only conformal symmetry. In Sect. 6, we resolve this question: Theorem 5 shows that data with apparent conformal symmetry always evolves to spacetimes with full isometries.

Before we state and prove our results, we review in Sect. 2 what globally hyperbolic spacetimes are and how they are described using the $3+1$ language; see, e.g., [9-11] for a more complete discussion. We then review how conformal and homothetic Killing vector fields, which are the infinitesimal generators of the corresponding spacetime symmetries, fit into the $3+1$ picture, following [12].

\section{Globally Hyperbolic Spacetimes and Conformal Symmetries}

Global hyperbolicity is a strong restriction on the structure of a spacetime ${ }^{1}$ $\left(\mathscr{M}^{4}, g\right)$. Explicitly, the condition may be stated as follows:

Definition 1. (Global Hyperbolicity and Cauchy Surfaces). A spacetime $\left(\mathscr{M}^{4}, g\right)$ is globally hyperbolic if there exists an embedded spacelike 3-manifold $\Sigma^{3}$ such that every endless causal curve intersects $\Sigma^{3}$ once and only once. Such a surface $\Sigma^{3}$, when it exists, is called a Cauchy surface.

Consequences of global hyperbolicity include the following:

a) $\mathscr{M}^{4}$ must be homotopic to $\Sigma^{3} \times \mathbf{R}$;

b) If $\tilde{\Sigma}^{3}$ is any compact spacelike 3 -manifold without boundary embedded in $\mathscr{M}^{4}$, then $\tilde{\Sigma}^{3}$ must (like $\Sigma^{3}$ ) be a Cauchy surface; and

c) if $g$ satisfies the vacuum Einstein equations, then it may be completely determined from a set of Cauchy data specified on $\Sigma^{3}$; or if $g$ satisfies the Einstein

1 Throughout, a spacetime consists of a smooth connected, orientable (and time-oriented) Hausdorff $C^{\infty}$ 4-manifold $\mathscr{M}^{4}$ and a smooth Lorentz-signature metric $g . C^{\infty}$ could easily be weakened to $C^{4}$ 
equations coupled to a well-posed hyperbolic system of matter equations, then the coupled system has the same property.

This last consequence is the key to many of the methods we use for proving our results below. It permits us to do essentially all of our analysis on a Cauchy surface $\Sigma^{3}$, which together with the pulled-back metric $\gamma$ constitutes a Riemannian manifold. The maximum principle and other techniques requiring elliptic operators may thus be used. In order to apply these techniques, we must first carry out the $3+1$ decomposition of the fields and the field equations: Starting with an embedding $i: \Sigma^{3} \hookrightarrow \mathscr{M}^{4}$, one defines

$$
\gamma_{a b}=i^{*} g_{a b} \text { intrinsic metric, }
$$

and

$$
K_{a b}=g\left(\nabla_{\hat{o}_{a}} e_{\perp}, \partial_{b}\right) \text { extrinsic curvature (or second fundamental form), }
$$

where $e_{\perp}$ is the vector field normal to $i\left(\Sigma^{3}\right)$, and $\partial_{a}$ and $\partial_{b}$ are vector fields tangent to $i\left(\Sigma^{3}\right)$. The information $\left(\gamma_{a b}, K_{c d}\right)$ constitutes a complete set of Cauchy data for the gravitational field on $i\left(\Sigma^{3}\right)$; if matter fields are present, they need their own Cauchy data. Here and below, Latin indices $a, b, c, \ldots m, n \ldots$ run over the spatial coordinates $1,2,3$, and Greek indices $\kappa, \lambda, \mu, v, \ldots$ run over the four spacetime indices $\perp, 1,2,3$; our conventions follow MTW [13] in general.

If we now choose an arbitrary vector field $\partial_{t}$ transverse to $i\left(\Sigma^{3}\right)$-with $3+1$ split $\partial_{t}=N e_{\perp}+M^{a} \partial_{a}$ - then the usual Gauss-Codazzi-Mainardi calculation performed on the spacetime curvature permits one to write out the Einstein equations

$$
G_{\mu \nu}=8 \pi T_{\mu \nu}
$$

in terms of $\gamma, K, N$, and $M$, as follows:

$$
\begin{gathered}
R+(\operatorname{tr} K)^{2}-K^{m}{ }_{n} K^{n}{ }_{m}=16 \pi T_{\perp \perp}, \\
\nabla_{a} K^{a}{ }_{b}-\nabla_{b}(\operatorname{tr} K)=-8 \pi T_{\perp b}, \\
\tilde{\mathscr{L}}_{\partial_{t}} \gamma_{a b}=-2 N K_{a b}+\tilde{\mathscr{L}}_{M} \gamma_{a b}, \\
\tilde{\mathscr{L}}_{\partial_{\mathrm{t}}} K^{a}{ }_{b}=N\left(R_{b}^{a}+(\operatorname{tr} K) K^{a}{ }_{b}\right)-\nabla^{a} \nabla_{b} N+\mathscr{L}_{M} K^{a}{ }_{b} \\
-8 \pi N\left[\frac{1}{2} \delta^{a}{ }_{b}\left(T^{m}{ }_{m}-T_{\perp \perp}\right)-T^{a}{ }_{b}\right] .
\end{gathered}
$$

Here $\nabla_{a}$ is the surface-intrinsic covariant derivative compatible with $\gamma_{a b}, R_{c d}$ is the corresponding surface-intrinsic Ricci curvature with scalar curvature $R$, and $\tilde{\mathscr{L}}$ is the surface-projected Lie derivative, defined in Appendix B. The first pair, Eqs. $(2.4,2.5)$, are constraints restricting the values of $\gamma_{a b}$ and $K_{d}^{c}$ on $i\left(\Sigma^{3}\right)$. The second pair, Eqs. $(2.6,2.7)$, are evolution equations describing how $\gamma_{a b}$ and $K_{d}^{c}$ evolve into the future (or the past) along $\partial_{t}$.

The mean curvature of the embedded hypersurface $i\left(\Sigma^{3}\right)$ is given by $\operatorname{tr} K$, which we shall denote by $\tau:=\operatorname{tr} K$. Hence a constant mean curvature (CMC) hypersurface is one with $\nabla_{a} \tau=0$ everywhere on it. As noted above, it is believed that "most spacetimes" contain CMC hypersurfaces. For later purposes, we find it useful to work with a trace decomposition of $K_{b}^{a}$, and write $K^{a}{ }_{b}=L_{b}^{a}+\frac{1}{3} \gamma^{a}{ }_{b} \tau$. Then Eqs. (2.4)-(2.7) translate into 


$$
\begin{aligned}
& R+\frac{2}{3} \tau^{2}-L^{m}{ }_{n} L^{n}{ }_{m}=16 \pi T_{\perp \perp}, \\
& \nabla_{a} L_{b}^{a}=\nabla_{b} \tau-8 \pi T_{\perp b} \\
& \tilde{\mathscr{L}}_{\partial_{t}} \gamma_{a b}=-2 N L_{a b}-\frac{2}{3} N \gamma_{a b} \tau+\tilde{\mathscr{L}}_{M} \gamma_{a b} \text {, } \\
& \widetilde{\mathscr{L}}_{\partial t} \tau=N\left(L^{m}{ }_{n} L^{n}{ }_{m}+4 \pi\left(T_{\perp \perp}+T^{m}{ }_{m}\right)+\frac{1}{3} \tau^{2}\right)-\nabla^{2} N+\widetilde{\mathscr{L}}_{{ }_{M}} \tau, \\
& \widetilde{\mathscr{L}}_{\partial_{t}} L^{a}{ }_{b}=N\left(\left(R^{a}{ }_{b}-\frac{1}{3} \gamma^{a}{ }_{b} R\right)+\tau L^{a}{ }_{b}-8 \pi\left(T^{a}{ }_{b}-\frac{1}{3} \gamma^{a}{ }_{b} T^{m}{ }_{m}\right)\right) \\
& -\left(\nabla^{a} \nabla_{b} N-\frac{1}{3} \gamma_{b}^{a} \nabla^{2} N\right)+\tilde{\mathscr{L}}_{M} L^{a}{ }_{b} .
\end{aligned}
$$

Let us now consider a one parameter group of conformal isometries in the spacetime $\left(\mathscr{M}^{4}, g\right)$, as represented by a spacetime conformal Killing vector field (CKV) $\xi$ :

$$
\mathscr{L}_{\xi} g_{\mu \nu}=\sigma g_{\mu \nu} .
$$

From (2.8) one easily derives

$$
\begin{aligned}
& \tilde{\mathscr{L}}_{\xi} \gamma_{a b}=\sigma \gamma_{a b}, \\
& \tilde{\mathscr{L}}_{\xi} L^{a}{ }_{b}=-\frac{1}{2} \sigma L^{a}{ }_{b},
\end{aligned}
$$

and

$$
\tilde{\mathscr{L}}_{\xi} \tau=-\frac{1}{2} \sigma \tau-\frac{3}{2} \nabla_{e_{\perp}} \sigma
$$

which describe how the $3+1$ data act under (projected) transport along $\xi$ [which need not be tangent to $i\left(\Sigma^{3}\right)$ ]. A further calcul tion [based on (2.9); see Appendix B] produces

$$
\tilde{\mathscr{L}}_{\xi} R_{b c d}^{a}=\frac{1}{2}\left(\gamma_{[d}^{a} \nabla_{c]} \nabla_{b} \sigma+\gamma_{b[d} \nabla_{c]} \nabla^{a}\right) \sigma
$$

from which one easily obtains $\tilde{\mathscr{L}}_{\xi} R_{b d}$ and $\tilde{\mathscr{L}}_{\xi} R$.

The most convenient way in which to simultaneously exploit the Einstein equations $\left(2.4^{\prime}\right)-\left(2.7^{\prime}\right)$ and the conformal isometry conditions (2.9)-(2.11) is to use the following trick (due to Berger [12]): We set the time evolution vector field $\partial_{t}$ of Eqs. $\left(2.6^{\prime}\right)-\left(2.7^{\prime}\right)$ equal to the CKV $\xi$. Thus the Einstein evolution equations $\left(2.6^{\prime}\right)-$ (2.7') may be combined wth the CKV evolution equations (2.9)-(2.11) to give us

$$
\begin{aligned}
& \sigma \gamma_{a b}=-2 \rho L_{a b}-\frac{2}{3} \rho \gamma_{a b} \tau+\tilde{\mathscr{L}}_{X} \gamma_{a b}, \\
& -\frac{1}{2} \sigma L_{b}^{a}=\rho\left(\left(R_{b}^{a}-\frac{1}{3} \gamma_{b}^{a} R\right)+\tau L_{b}^{a}-8 \pi\left(T_{b}^{a}-\frac{1}{3} \gamma_{b}^{a} T^{m}{ }_{m}\right)\right) \\
& -\left(\nabla^{a} \nabla_{b} \rho-\frac{1}{3} \gamma^{a}{ }_{b} \nabla^{2} \rho\right)+\tilde{\mathscr{L}}_{X} L_{b}{ }_{b},
\end{aligned}
$$

and

$$
-\frac{1}{2} \sigma \tau-\frac{3}{2} \nabla_{e_{\perp}} \rho=\rho\left(L^{m}{ }_{n} L_{m}^{n}+4 \pi\left(T_{\perp \perp}+T_{m}^{m}\right)+\frac{1}{3} \tau^{2}\right)-\nabla^{2} \rho+\tilde{\mathscr{L}}_{X} \tau,
$$

where we have used the $3+1$ decomposition

$$
\xi=\sigma e_{\perp}+X
$$

[ $X$ tangent to $i\left(\Sigma^{3}\right)$ ] to distinguish this particular choice of $\partial_{t}$ from the generic choice. These Eqs. (2.13)-(2.15) play an important role in the proof of several of our theorems. 
The results of this paper require either the vacuum Einstein equation (2.3) without cosmological constant, or the Klein-Gordon or Yang-Mills equations coupled to the Einstein equations, or an energy condition on the stress tensor $T$. Two energy conditions will be used:

Definition 2. (Mixed Energy Condition). A stress energy tensor $T$ is said to obey the Mixed Energy Condition if at any point $x$ on any hypersurface,

a) the Strong Energy Condition holds,

$$
T_{\perp \perp}+\left.T_{m}^{m}\right|_{x} \geqq 0 ; \text { and furthermore }
$$

b) equality in a) implies that all components of $T$ are zero,

$$
T_{\perp \perp}+\left.T_{m}^{m}\right|_{x}=\left.0 \Rightarrow T_{\mu \nu}\right|_{x}=0 .
$$

The Mixed Energy Condition is a slightly stronger form of the Strong Energy Condition ([14], p. 95). For example, a perfect fluid with energy density $\mu \geqq 0$ and pressure $p$ will obey the Mixed Energy Condition as long as $p>-\mu / 3$ everywhere.

Definition 3. (Dominant Energy Condition). A stress energy tensor $T$ is said to obey the Dominant Energy Condition $([14], p .91)$ if at any point $x$ on any hypersurface

$$
T_{\perp \perp} \geqq\left|T_{\hat{\mu} \hat{\mu}}\right|
$$

where $T_{\hat{\mu} \hat{\mu}}$ is any component of the stress energy tensor in any orthonormal frame.

Under the Dominant Energy Condition, if $T$ vanishes on a Cauchy surface, then it vanishes throughout spacetime $([14]$, p. 94). (The hypothesis can actually be weakened to $T_{\perp \perp} \geqq c \mid T_{\hat{\mu} \hat{\mu} \mid}$ for some positive constant $c$, but there is little reason to do so because the Dominant Energy Condition holds in all interesting applications.)

\section{Homothetic Symmetries of Spatially Compact Spacetimes}

Many solutions of Einstein's equations for vacuum or for various forms of matter are known, which admit a proper homothetic Killing vector field; see e.g., [15]. (A proper homothetic Killing field is one which is not just Killing.) However, none of these known solutions are spatially compact. In this theorem we assume spatial compactness; we further must make the technical assumption that spacetime admits a hypersurface of constant mean (extrinsic) curvature. We show that essentially no solutions exist.

Theorem 1. (Homothetic Symmetries of Spatially Compact Einstein Solutions). Let $\left(\mathscr{M}^{4}, g\right)$ be a globally hyperbolic spacetime which

a) satisfies the Einstein equations for a stress tensor T obeying the Mixed Energy Condition and the Dominant Energy Condition;

b) admits a homothetic Killing vector field $\xi$ of $g$; and

c) admits a compact hypersurface of constant mean curvature.

Then either $\left(\mathscr{M}^{4}, g\right)$ is an expanding hyperbolic model with metric (1.1) with $T$ vanishing everywhere, or $\xi$ is a Killing vector field.

Proof. We work on the compact hypersurface $i\left(\Sigma^{3}\right)$ of constant mean curvature and use the $3+1$ split. Thus $\tau$ and $\sigma$ are constant, and we shall assume $\sigma \neq 0$, for if $\sigma=0$, 
then $\xi$ is a Killing vector field and we are done. The trace of (2.3) is

$$
3 \sigma=-2 \rho \tau+2 \nabla_{a} X^{a} .
$$

Integrating (3.1) over the hypersurface $i\left(\Sigma^{3}\right)$ gives

$$
3 \sigma V=-2 \tau \int_{i\left(\Sigma^{3}\right)} \rho
$$

where $V$ is the volume of $i\left(\Sigma^{3}\right)$ and where the divergence term involving $X^{a}$ vanishes by the divergence theorem. In (3.2) the left-hand side is nonzero; hence $\tau \neq 0$.

Putting $\tau=$ const in (2.15) we obtain an elliptic equation which $\rho$ must satisfy,

$$
-\frac{1}{2} \sigma \tau=\left(-\nabla^{2}+U\right) \rho
$$

where

$$
U \equiv L_{b}^{a} L_{a}^{b}+\frac{1}{3} \tau^{2}+4 \pi\left(T_{\perp \perp}+T_{m}^{m}\right) .
$$

We first observe that $U>0$ on $i\left(\Sigma^{3}\right)$ by the Mixed Energy Condition (see Definition 2) and by the facts that $\tau \neq 0$ and $L^{a}{ }_{b} L^{b}{ }_{a} \geqq 0$. Since the elliptic operator $\left(-\nabla^{2}+U\right)$ is positive, and since the left-hand side of $(3.3 \mathrm{a})$ is constant, we expect that $\rho$ is strictly bounded away from 0 on $i\left(\Sigma^{3}\right)$ and is opposite in sign to $\sigma \tau$. This expectation is proven by the following standard argument. For definiteness, consider the case $\sigma \tau<0$. At a point $x \in i\left(\Sigma^{3}\right)$ where $\rho$ achieves its global minimum, $\nabla^{2} \rho(x) \geqq 0$; then (3.3a) implies that $U \rho(x)>0$. Since $U(x)>0, \rho(x)>0$ at its global minimum; hence $\rho>0$ everywhere. A similar argument shows that if $\sigma \tau>0$, then $\rho<0$ everywhere.

Integrating (3.3a) over $i\left(\Sigma^{3}\right)$, the term $-\nabla^{2} \rho$ vanishes by the divergence theorem, and we obtain

$$
-\frac{1}{2} \sigma \tau V=\int_{i\left(\Sigma^{3}\right)} U \rho
$$

Now multiply (3.2) by $\tau / 6$ and add to (3.4). The result is

$$
0=\int_{i\left(\Sigma^{3}\right)}\left(U-\frac{1}{3} \tau^{2}\right) \rho=\int_{i\left(\Sigma^{3}\right)}\left[L^{a}{ }_{b} L_{a}^{b}+4 \pi\left(T_{\perp \perp}+T^{m}{ }_{m}\right)\right] \rho .
$$

Since the integrand in (3.5) is everywhere $\geqq 0$ (respectively $\leqq 0$ ) if $\sigma \tau<0$ (respectively $\sigma \tau>0$ ) it must vanish everywhere. Since $\rho$ is everywhere nonzero, the two terms in [ ] must vanish; they are individually nonnegative, and so

$$
\begin{aligned}
L_{b}^{a} & \equiv 0, \\
T_{\perp \perp}+T_{m}^{m} & \equiv 0
\end{aligned}
$$

everywhere on $i\left(\Sigma^{3}\right)$. Thus $U=\tau^{2} / 3=$ const and the unique solution $\rho$ of (3.3a) is

$$
\rho=-\frac{3 \sigma}{2 \tau}=\text { const. }
$$

From (3.6),

$$
K_{a b}=\frac{1}{3} \tau \gamma_{a b}
$$


The Mixed Energy Condition and (3.7) imply that

$$
T_{\mu \nu} \equiv 0 \quad \text { on } i\left(\Sigma^{3}\right) \text {. }
$$

Using (3.6), (3.8) and (3.10) in (2.14), we find that Ricci tensor of $\gamma$ is a pure constant trace,

$$
\begin{aligned}
R_{a b} & =\frac{1}{3} R \gamma_{a b} \\
& =-\frac{2 \tau^{2}}{9} \gamma_{a b},
\end{aligned}
$$

where (3.6) and (3.7) were used in $\left(2.4^{\prime}\right)$ to evaluate $R$. For a 3-dimensional Riemannian space, the Riemann tensor is determined by the Ricci tensor; thus (3.11a) implies that the metric $\gamma$ is the metric of a space of constant negative curvature. Only the standard hyperbolic metrics satisfy this condition, and they are specified by the choice of global topology and the choice of a single scale factor.

Substituting (3.6) and (3.8) into (2.13), we find that $X^{a}$ is a Killing vector of the 3geometry. The standard hyperbolic metrics admit no globally defined Killing vector fields [16]. Therefore $X^{a}$ vanishes, so the homothetic Killing vector field $\xi$ is orthogonal to $i\left(\Sigma^{3}\right)$.

The allowable initial data for a globally hyperbolic spacetime satisfying our various hypotheses and having a proper homothetic symmetry is now determined to be of the form $\left(\gamma_{a b}=9 h_{a b} / \tau^{2}, K_{a b}=\frac{1}{3} \gamma_{a b} \tau\right)$ for some nonzero constant $\tau$, with $T_{\mu \nu} \equiv 0$. Here $h_{a b}$ is a metric of constant negative curvature with curvature scalar $R=-6$. From the Dominant Energy Condition, $T_{\mu \nu} \equiv 0$ throughout spacetime, and then it is easily checked that the vacuum spacetime development of the initial data is necessarily of the form (1.1), thus completing the proof.

As an interesting example, Theorem 1 applies directly to the Einstein-YangMills theory [17], with the gauge group chosen to a compact Lie group. The fields in this theory are the spacetime metric $g_{\mu \nu}$ and the Lie-algebra-valued gauge potential $A_{\mu}$. If one then defines the Lie-algebra-valued Yang-Mills field strength as

$$
F_{\mu \nu}=D_{\mu} A_{\nu}-D_{v} A_{\mu}+\left[A_{\mu}, A_{\nu}\right]
$$

where $D_{\mu}$ is the usual spacetime covariant derivative operator (based on $g_{\mu v}$ ), and if [,] is the bracket in the Lie algebra, then the Einstein-Yang-Mills field equations are

$$
\begin{aligned}
G_{\mu \nu} & =8 \pi T_{\mu \nu}, \\
D^{\lambda} F_{\lambda \mu}+\left[A^{\lambda}, F_{\lambda \mu}\right] & =0,
\end{aligned}
$$

where the Yang-Mills stress-energy tensor is

$$
T_{\mu \nu}=\frac{1}{4 \pi}\left(F_{\mu \lambda} \cdot F_{\nu}{ }^{\lambda}-\frac{1}{4} g_{\mu \nu} F_{\lambda \kappa} \cdot F^{\lambda \kappa}\right)
$$

One easily verifies that the Mixed Energy Condition and the Dominant Energy Condition are satisfied by this theory; the calculation 


$$
T_{\perp \perp}+T_{m}^{m}=\frac{1}{4 \pi}\left(E_{a} \cdot E^{a}+B_{a} \cdot B^{a}\right)
$$

where $E_{a}=F_{\perp a}$, and $B^{a}=\frac{1}{2} \varepsilon^{\perp a b c} F_{b c}$, is useful in showing this. (Note that the inner product · in the Lie algebra can be, and is always, chosen so as to be positive definite.) Thus we have the following:

Corollary 1. (Homothetic Symmetries of Einstein-Yang-Mills Solutions). Let $(\mathscr{M}, g, A)$ be a globally hyperbolic spacetime which

a) satisfies the Einstein-Yang-Mills equations:

b) admits a homothetic Killing vector field $\xi$ of $g$ : and

c) admits a compact hypersurface of constant mean curvature.

Then either $\left(\mathscr{M}^{4}, g\right)$ is an expanding hyperbolic model with metric (1.1) with the field strength $F$ vanishing everywhere, or $\xi$ is a Killing vector field.

Three remarks are in order. First, $F$ vanishes because $T$ does; see (3.15). Second, $A$ need not vanish; but since $F$ vanishes, $A$ is, in mathematician's language, a "trivial connection," or in physicist's language, a "pure gauge field." Third, it was not actually necessary to check the Dominant Energy Condition; the vanishing of $T$ on a Cauchy surface implies that $F$ vanishes throughout spacetime simply because the Yang-Mills equations are a well posed hyperbolic system.

As a special case, Corollary 1 applies to the Einstein-Maxwell equations (see Hawking and Ellis [14], p. 68).

A slightly different case is that of a massless scalar field $\psi$ coupled to gravity, the massless Einstein-Klein-Gordon equations. Here $g_{\mu \nu}$ and $\psi$ obey the equations

$$
\begin{aligned}
D^{\mu} D_{\mu} \psi & =0, \\
G_{\mu \nu} & =8 \pi T_{\mu \nu},
\end{aligned}
$$

with stress tensor

$$
T_{\mu \nu}=\partial_{\mu} \psi \partial_{\nu} \psi-\frac{1}{2} g_{\mu \nu} \partial^{\lambda} \psi \partial_{\lambda} \psi
$$

The massless scalar field does not obey the Mixed Energy Condition; rather,

$$
T_{\perp \perp}+T_{m}^{m}=2\left(\partial_{e_{\perp}} \psi\right)^{2} .
$$

If at a point the left-hand side in (3.18) is zero, then $\partial_{e_{\perp}} \psi=0$ there but we get no information about $\nabla_{a} \psi$, and $\psi$ restricted to $i\left(\Sigma^{3}\right)$ could, roughly speaking, be anything. In this case we need to slightly modify the proof of Theorem 1 .

Theorem 2. (Homothetic Symmetries of Einstein-Klein-Gordon Solutions). Let $\left(\mathscr{M}^{4}, g\right)$ be a globally hyperbolic spacetime which

a) satisfies the Einstein-Klein-Gordon equations;

b) admits a homothetic Killing vector field $\xi$ of $g$; and

c) admits a compact hypersurface of constant mean curvature.

Then either $\left(\mathscr{M}^{4}, g\right)$ is an expanding hyperbolic model with metric (1.1) and $\psi$ is constant everywhere, or $\xi$ is a Killing vector field.

Proof. We follow the proof of Theorem 1 exactly through (3.9). However, instead of 
(3.10) we have only

$$
\begin{aligned}
\partial_{e_{\perp}} \psi & \equiv 0, \\
T_{\perp \perp} & =\frac{1}{2} \nabla^{a} \psi \nabla_{a} \psi, \\
T_{a}^{\perp} & \equiv 0, \\
T_{a b} & =\nabla_{a} \psi \nabla_{b} \psi-\frac{1}{2} \gamma_{a b} \nabla^{c} \psi \nabla_{c} \psi .
\end{aligned}
$$

Using (3.6), (3.8), (3.9), (3.20) and (3.22) we deduce from (2.14) and (2.4') that the Ricci tensor of $\gamma$ is

$$
R_{a b}=8 \pi \nabla_{a} \psi \nabla_{b} \psi-\frac{2 \tau^{2}}{9} \gamma_{a b}
$$

(cf. 3.11b). The contracted Bianchi identity now gives

$$
0=\nabla_{b} R_{a}^{b}-\frac{1}{2} \nabla_{a} R=8 \pi \nabla_{a} \psi \nabla^{2} \Psi .
$$

This equation implies that the scalar field $\psi$ is constant on $i\left(\Sigma^{3}\right)$. For $\nabla^{2} \psi$ vanishes outside the support of $\nabla_{a} \psi$, and by (3.24) it also vanishes inside the support of $\nabla_{a} \psi$. Therefore $\nabla^{2} \psi=0$ on $i\left(\Sigma^{3}\right)$ and $\psi=$ const on $i\left(\Sigma^{3}\right)$. Thus the massless wave (3.16a) for $\psi$ has trivial Cauchy data $\left(\psi, \partial_{e_{1}} \psi\right)=($ const, 0$)$ on the Cauchy surface $i\left(\Sigma^{3}\right)$. Therefore $\psi=$ const throughout $M^{4}$.

The massless Klein-Gordon equation admits the global symmetry $\psi \rightarrow$ $\psi+$ const. Therefore a constant solution is equivalent to a vanishing solution. The pure constant solutions $\psi$ to the Klein-Gorden equation are analogous to the pure gauge solutions $A$ to the Yang-Mills equations.

We would like to do away with hypothesis c) of Theorems 1 and 2, which assumes a constant mean curvature (CMC) hypersurface, but we have been unable to do so completely. We mention a partial result:

Proposition 1. Let $\left(\mathscr{M}^{4}, g\right)$ be a globally hyperbolic spacetime which

a) satisfies the Einstein equations for a stress tensor T obeying the Mixed Energy Condition and the Dominant Energy Condition;

b) admits a homothetic Killing vector field $\xi$ of $g$;

c) admits a Cauchy surface i( $\left.\Sigma^{3}\right)$ such that the normal component $\rho$ of $\xi$ [cf. (2.16)] is everywhere nonvanishing, and is thus of the same sign on $i\left(\Sigma^{3}\right)$, say $\rho>0$.

Then either $\left(\mathscr{M}^{4}, g\right)$ is an expanding hyperbolic model with metric (1.1) with $T$ vanishing everywhere, or $\xi$ is a Killing vector field.

Sketch of Proof. The domain of development of $i\left(\Sigma^{3}\right)$ can be exhibited explicitly as a manifold $\mathscr{M}^{4}=\Sigma^{3} \times \mathbf{R}$ with metric $g$ given by

$$
d s^{2}=e^{\sigma t}\left(-\left(\rho^{2}-X_{a} X^{a}\right) d t^{2}+2 X_{a} d x^{a} d t+\gamma_{a b} d x^{a} d x^{b}\right),
$$

where $\rho, X^{a}$, and $\gamma_{a b}$ are functions of the spatial coordinates $\left\{x^{a}\right\}$ but not of the time coordinate $t$; here the $\left\{x^{a}\right\}$ are coordinates on $\Sigma^{3}$ and $t \in \mathbf{R}$. The hypersurface $t=0$ is just $i\left(\Sigma^{3}\right)$, which fixes $\rho, X^{a}$, and $\gamma_{a b}$.

Our goal is to show that a CMC hypersurface exists in $\left(\mathscr{M}^{4}, g\right)$. To do this we use an existence result of C. Gerhardt ([1], Theorem 5.2). First, consider the special case 
$X^{a} \equiv 0$. Gerhardt's result applies directly and a CMC surface exists. In the general case, we can carry out a coordinate transformation of the form $t^{\prime}=t, x^{\prime a}=x^{\prime a}\left(t, x^{a}\right)$ which will set the off-diagonal components of the metric $g^{\prime}{ }_{0 a} \equiv 0$; i.e., $X^{\prime}{ }_{a} \equiv 0$ in the new coordinate system. This leaves $\rho^{\prime}$ and $\gamma_{a b}^{\prime}$ time-dependent but still well behaved on compact sets in $\left(\mathscr{M}^{4}, g\right)$. Then Gerhardt's result applies to the general case as well and a CMC hypersurface exists. We use Theorem 1 above to finish the proof.

\section{Proper Conformal Symmetries of Vacuum Spacetimes}

We now examine the case of proper conformal Killing vectors $\xi$. A proper conformal symmetry is one which is not just a homothetic symmetry; i.e., the function $\sigma$ in (2.8) is not constant. Since many of the Friedmann-Robertson-Walker solutions admit several proper conformal Killing vectors, the obvious generalization of Theorem 1 does not hold. If we confine attention to the vacuum Einstein equations $\left(T_{\mu \nu}=0\right)$, however, we have a strong result that is completely local and does not depend on boundary conditions:

Theorem 3. (Conformal Symmetries of Vacuum Spacetimes). Let $\left(\mathscr{M}^{4}, g\right)$ be a spacetime which satisfies the vacuum Einstein equations and admits a conformal Killing vector field $\xi$ of $g$. Then either

a) $\left(\mathscr{M}^{4}, g\right)$ is everywhere locally flat; or

b) $\left(\mathscr{M}^{4}, g\right)$ is a "plane-fronted wave" with metric (1.2); or

c) $\xi$ is a homothetic Killing vector field.

Proof. As pointed out by Berger [12], the system of differential equations

$$
\begin{aligned}
\mathscr{L}_{\xi} g_{\mu \nu} & =\sigma g_{\mu \nu} & & \text { (conformal Killing equation), } \\
R_{\mu \nu} & =0 & & \text { (vacuum Einstein equation) }
\end{aligned}
$$

has nontrivial integrability conditions. Here $R_{\mu \nu}$ is the Ricci tensor of $g_{\mu \nu}$. (For homothetic or full Killing symmetries, the integrability conditions are satisfied identically.) The first of these conditions follows from the calculation (see, e.g., [18])

$$
\mathscr{L}_{\xi} R_{\mu \nu}=-D_{\mu} D_{v} \sigma-\frac{1}{2} g_{\mu \nu} D^{\lambda} D_{\lambda} \sigma .
$$

This, together with (4.1b), implies

$$
D_{\mu} D_{v} \sigma=0 .
$$

Then, taking another derivative of (4.2) and antisymmetrizing, we obtain a second integrability condition

$$
0=-2 D_{[\lambda} D_{\mu]} D_{v} \sigma=\left(D_{\rho} \sigma\right) R^{\rho}{ }_{\nu \lambda \mu}
$$

which, since $R_{\mu v}$ vanishes, may be rewritten as

$$
0=\left(D_{\rho} \sigma\right) C^{\rho}{ }_{\nu \lambda \mu},
$$

where $C^{\rho}{ }_{\nu \lambda \mu}$ is the Weyl curvature tensor of $g_{\mu v}$. Conditions (4.2) and (4.3) are sufficient to prove our results, although more integrability conditions may be derived.

As a consequence of (4.2), $D^{\mu} \sigma$ is a covariantly constant vector field, and 
$\left(D^{\mu} \sigma\right)\left(D_{\mu} \sigma\right)$ is a spacetime constant. Hence the character of the vector field $D^{a} \sigma-$ spacelike, timelike, lightlike, or zero - at one point is the same as at every point of spacetime. We assume that $D^{\mu} \sigma \neq 0$; otherwise $\sigma=$ const, in which case we have a homothetic Killing field. The following lemma is now useful:

Lemma 1. If $V^{\mu}$ is a timelike or spacelike vector, then the condition $V_{\rho} C^{\rho}{ }_{v \lambda \mu}=0$ implies that $C^{\rho}{ }_{v \lambda \mu}=0$.

This lemma is proven by a straightforward combinatorial argument, which we omit. It depends on the index symmetries of $C^{\rho}{ }_{\nu \lambda \mu}$, i.e., $C_{\rho \nu \lambda \mu}=-C_{\rho \nu \mu \lambda}=C_{\lambda \mu \rho \nu}=$ $-C_{\rho \lambda \mu \nu}-C_{\rho \mu \nu \lambda}$, on its tracelessness $C^{\rho}{ }_{v \rho \mu}=0$, and on the fact that the space orthogonal to $V^{\mu}$ is 3-dimensional.

It follows immediately from this lemma that if $D^{\mu} \sigma$ is timelike or spacelike, then the Riemann tensor vanishes and so spacetime is locally flat. This must be true everywhere in spacetime. If, on the other hand, the vector field $D^{\mu} \sigma$ is lightlike, then (4.2) implies that $D^{\mu} \sigma$ generates a nonrotating, shear free, divergence free, null geodesic congruence. From (4.3), the Weyl tensor is of Petrov type N, with principal null congruence $D^{\mu} \sigma$. Then it follows [4] that $\left(\mathscr{M}^{4}, g\right)$ is of the particular form of a "plane-fronted gravitational wave with parallel rays," with metric (1.2). We may choose $u \equiv \sigma$. Solutions do exist of this form; details are given in Appendix A.

Therefore, certain plane-fronted waves (1.2) and Minkowski spacetime are the only vacuum solutions of Einstein's equations with a proper conformal Killing vector. For the plane-fronted waves, the function $H$ must obey further complicated restrictions, which are discussed in Appendix A.

As a particular consequence of Theorem 3, and of Theorem 1, we find:

\section{Corollary 2. (Conformal Symmetries of Spatially Compact, Vacuum Einstein}

Solutions). Let $\left(\mathscr{M}^{4}, g\right)$ be a globally hyperbolic spacetime which

a) satisfies the vacuum Einstein equations

b) admits a homothetic Killing vector field $\xi$ of $g$; and

c) admits a compact hypersurface of constant mean curvature.

Then either $\left(\mathscr{M}^{4}, g\right)$ is an expanding hyperbolic model with metric (1.1) and $\xi$ is a homothetic Killing vector field, or $\xi$ is a Killing vector field.

To verify this corollary one has to know that alternatives a) and b) to the conclusion of Theorem 3 do not admit spatial compactification; this is a nontrivial fact. Rather than interrupting our line of argument to prove this fact, we present in Appendix C a direct proof of Corollary 2.

What about the Einstein equations coupled to the Klein-Gordon equation or the Yang-Mills equations? The Einstein-Klein-Gordon equations clearly admit spatially compact $(k=+1)$ Friedmann-Robertson-Walker solutions with spatially homogeneous but time dependent $\psi$. The Einstein-Yang-Mills equations also admit $k=+1$ Friedmann-Robertson-Walker solutions, as is shown in Appendix D. Since these solutions generally admit nine proper conformal Killing vector fields, the obvious generalization of Theorem 3 is false for these systems of equations.

Some other interesting recent work related to the questions raised here is that of Garfinkel and Tiem [19]. They show, without having to impose any boundary conditions, that the only spacetime satisfying the vacuum Einstein equations with 
nonzero cosmological constant and admitting a proper conformal Killing field is de Sitter spacetime.

It is not clear to us what the right conjecture is, on the subject of solutions of Einstein's equations with matter having proper conformal symmetry. Any conjectures the reader would like to frame should take into account the examples of this section and of Appendices A and C.

\section{Conformal Symmetries of Asymptotically Flat Spacetimes}

We have shown that there is essentially only one spatially compact, globally hyperbolic spacetime solution of the Einstein equations that admits a proper homothetic symmetry (admitting a hypersurface of constant mean extrinsic curvature, and under certain energy conditions, in particular for the EinsteinYang-Mills and massless Einstein-Klein-Gordon equations). That solution is trivial in that it is Minkowski spacetime identified in some fashion. Likewise we have shown that vacuum spacetimes that admit proper conformal symmetries are extremely rare.

What if we consider spacetimes which are asymptotically flat, or have other boundary conditions? In the asymptotically flat case, we have a strong result.

Theorem 4. (Conformal Symmetries of Asymptotically Flat Einstein Solutions). Let $\left(\mathscr{M}^{4}, g\right)$ be a globally hyperbolic spacetime which

a) is spatially asymptotically flat, with $\mathscr{M}^{4}=R^{4}$;

b) satisfies the Einstein equations for a stress tensor $T$ obeying the Dominant Energy Condition, with $T$ asymptotically $O\left(r^{-4}\right)$; and

c) admits a conformal Killing field $X$ which asymptotically approaches the dilation vector field

$$
\xi_{\text {dilat }}=x^{\mu} \partial_{\mu}
$$

(in appropriate asymptotic coordinates). Then $\left(\mathscr{M}^{4}, g\right)$ is Minkowski spacetime.

Proof. The proof is "easy" and completely "local at infinity," though it relies on the positive energy theorem, a deep and global result. To be specific about the asymptotic conditions, we assume that $\left(\mathscr{M}^{4}, g\right)$ admits a coordinate system $\left\{x^{\mu}\right\}$ such that

$$
g_{\mu \nu}=\eta_{\mu \nu}+O\left(r^{-1}\right)
$$

and in particular

$$
g_{a b}=\left(1+\frac{2 M}{r}\right) \delta_{a b}+O\left(r^{-2}\right) ;
$$

and also that the first derivatives of (5.2) and (5.3) hold, e.g.,

$$
\begin{aligned}
& \partial_{c} g_{a b}=-\frac{2 M x^{c}}{r^{3}} \delta_{a b}+O\left(r^{-3}\right), \\
& \partial_{0} g_{a b}=O\left(r^{-2}\right) .
\end{aligned}
$$


We also assume that in a similar way the second derivatives of (5.3) hold as $r \rightarrow \infty$; this hypothesis is necessary for the positive energy theorem. Here $\eta_{\mu \nu} \equiv \operatorname{diag}(-1$, $+1,+1,+1)$ is the metric of Minkowski spacetime, the constant $M$ is the ADM mass of the spacetime, and $r \equiv\left(x^{a} x^{a}\right)^{1 / 2}$.

Under these conditions and under the Dominant Energy Condition, the positive energy theorem holds [20-22]: $M \geqq 0$, and $M=0$ only if $\left(\mathscr{M}^{4}, g\right)$ is Minkowski spacetime. Now choose a particular time slice, for instance the slice $x^{0}=0$, and work in this slice; then (5.4) implies $K_{a b}=O\left(r^{-2}\right)$. For $\xi=\rho e_{\perp}+X$ as in (2.8), we specify the asymptotic conditions c) as $r \rightarrow \infty$ (for fixed $x^{0}$ ) in the form

$$
\begin{aligned}
\rho & =c+O\left(r^{-1}\right), \\
X^{a} & =x^{a}+f^{a}\left(x^{c}\right)+O\left(r^{-1}\right) ;
\end{aligned}
$$

here $c$ is a constant and $f$ is a function which is homogeneous of degree 0 in the spatial coordinates $\left\{x^{c}\right\}$; roughly speaking, $c$ and $x^{a}$ are the flat spacetime pieces of $\xi$, and arbitrary corrections appear at the next order in $1 / r$. We further assume that the first derivatives of (5.5) are well behaved. From (5.4) and (5.5),

$$
\sigma=2+O\left(r^{-1}\right)
$$

where $\sigma \equiv \frac{1}{2} \nabla_{\mu} \xi^{\mu}$ as in Sect. 2. Inserting (5.2), (5.3), (5.4) and (5.5) into (2.13) gives

$$
\begin{aligned}
\sigma\left(1+\frac{2 M}{r}\right) \delta_{a b} & =\tilde{\mathscr{L}}_{x^{c}}\left[\left(1+\frac{2 M}{r}\right) \delta_{a b}\right]+\tilde{\mathscr{L}}_{f^{c}} \delta_{a b}+O\left(r^{-2}\right) \\
& =\delta_{a b} \widetilde{\mathscr{L}}_{x^{c}}\left(1+\frac{2 M}{r}\right)+\left(1+\frac{2 M}{r}\right) \tilde{\mathscr{L}}_{x^{c}} \delta_{a b}+\tilde{\mathscr{L}}_{f^{c}} \delta_{a b}+O\left(r^{-2}\right) \\
& =-\frac{2 M}{r} \delta_{a b}+2\left(1+\frac{2 M}{r}\right) \delta_{a b}+\partial_{a} f_{b}+\partial_{b} f_{a}+O\left(r^{-2}\right) .
\end{aligned}
$$

Contracting (5.7) without $x^{a} x^{b} / r^{2}$ eliminates the terms in $f$ thanks to homogeneity: $x^{c} \partial_{c} f^{a}=0$; and gives

$$
\sigma\left(1+\frac{2 M}{r}\right)=-\frac{2 M}{r}+2\left(1+\frac{2 M}{r}\right)+O\left(r^{-2}\right)
$$

or

$$
\sigma=2-\frac{2 M}{r}+O\left(r^{-2}\right)
$$

Following assumption b) stated in the hypotheses of this theorem, we assume falloffs of $T$ such that

$$
\begin{aligned}
T_{\mu \nu} & =O\left(r^{-4}\right), \\
\partial_{\lambda} T_{\mu \nu} & =O\left(r^{-5}\right) .
\end{aligned}
$$

Using the Einstein equations $R_{\mu \nu}=T_{\mu \nu}-\frac{1}{2} g_{\mu \nu} T$ together with (4.2), and from the fall-off rates (5.2), (5.3), (5.5), (5.9) we have assumed, we find

$$
D_{\mu} D_{v} \sigma=\mathscr{L}_{\xi}\left(T_{\mu \nu}-\frac{1}{6} g_{\mu \nu} T\right)=O\left(r^{-4}\right)
$$


and in particular for the $a b$ components, using (5.8),

$$
\frac{6 x^{a} x^{b} / r^{2}-2 \delta_{a b}}{r^{3}} M=O\left(r^{-4}\right)
$$

Thus $M=0$, and so by the positive energy theorem, $\left(\mathscr{M}^{4}, g\right)$ is Minkowski spacetime.

Yip [23] has also done calculations recently on the asymptotically flat case.

We remark that spacetimes $d o$ exist which are asymptotically flat at null infinity $\mathscr{I}^{+}$, and which admit a homothetic symmetry asymptotic to a dilatation (5.1), though they fail to the asymptotically flat at spatial infinity in accord with Theorem 4. Such solutions have a Bondi-Sachs mass $M_{\mathrm{BS}}(u)$ which is linear in retarded time $u$ on $\mathscr{I}^{+}$,

$$
M_{\mathrm{BS}}(u)=a u+b, \quad a=\mathrm{const} \neq 0, b=\mathrm{const}
$$

this linear behavior is forced by scale invariance. The ADM mass should be the limit of $M_{\mathrm{BS}}(u)$ as $u \rightarrow-\infty$, but this limit is infinite, which indicates that the spacetime is not well behaved at spatial infinity. An example of such a solution to the massless Einstein-Klein-Gordon equations (3.16) is given in [24].

\section{The York Map and Linearization Instability}

As noted in the introduction, one of the motivations for our work on homothetic and conformal isometries stems from a question which arises in the analysis of the Lichnerowicz-Choquet Bruhat-York (LCY) procedure for solving the constraint equations of general relativity. We will state this question and resolve it here, but first we provide a quick sketch of how the LCY procedure works. (A more complete discussion appears in Choquet-Bruhat and York [11].) Note that, although the LCY procedure can be applied to the Einstein equations (2.3) coupled to nongravitational fields, we treat only the vacuum case $T_{\mu \nu}=0$.

The goal is to obtain data $(\gamma, K)$ on some given 3-manifold $\Sigma^{3}$ which satisfy the constraint equations (2.4) and (2.5) (with $T_{\mu \nu}=0$ ). The LCY method derives such data from certain "free data" via the following steps:

1) Choose an arbitrary Riemannian metric $\lambda_{a b}$ and an arbitrary symmetric tensor $\mu^{c d}$ such that $\bar{\nabla}_{c} \mu^{c d}=0$ (transverse) and $\lambda_{c d} \mu^{c d}=0$ (traceless). [We denote by " $T_{T T}^{*} \mathscr{M}\left(\Sigma^{3}\right)$ " the set of all such $\left(\lambda_{a b}, \mu^{c d}\right)$; note that $\bar{\nabla}$ is the covariant derivative compatible with $\lambda_{a b}$.]

2) Pick an arbitrary constant $\tau$. [This will be the mean curvature of the data $(\gamma, K)$; note that the LCY procedure only produces constant mean curvature data.]

3) Solve the quasilinear elliptic equation

$$
\bar{\nabla}^{2} \varphi=\frac{1}{8} \bar{R} \varphi-\frac{1}{8} \mu_{d}^{c} \mu_{c}^{d} \varphi^{-7}+\frac{1}{12} \tau^{2} \varphi^{5}
$$

for $\varphi$. (Here $\bar{\nabla}$ and $\bar{R}$ are based on $\lambda_{a b}$, and the indices of $\mu_{d}^{c}$ are lowered using $\lambda_{a b}$.)

4) Construct

$$
\gamma_{a b}=\varphi^{4} \lambda_{a b}
$$


and

$$
K^{a b}=\varphi^{-10} \mu^{a b}+\frac{1}{3} \varphi^{-4} \lambda^{a b} \tau .
$$

For an open dense subset $\mathscr{A}\left(\Sigma^{3}\right) \subset T_{T T}^{*} \mathscr{M}\left(\Sigma^{3}\right)$, Eq. (6.1) can be solved uniquely. ${ }^{2}$ If we use $\mathscr{C}\left(\Sigma^{3}\right)$ to denote the data $(\gamma, K)$ which satisfy the constraints, we may view the LCY procedure as a map (the "York map")

$$
\mathscr{Y}_{\tau}: \mathscr{A}\left(\Sigma^{3}\right) \rightarrow \mathscr{C}\left(\Sigma^{3}\right) \text {. }
$$

This map has many nice properties (see Isenberg and Marsden [26] for a discussion of some of them). Of primary concern here is how it behaves relative to the singular points of $\mathscr{A}\left(\Sigma^{3}\right)$ and $\mathscr{C}\left(\Sigma^{3}\right)$.

Both $\mathscr{A}\left(\Sigma^{3}\right)$ and $\mathscr{C}\left(\Sigma^{3}\right)$ are "stratified manifolds" with well-understood singular point structure. $\mathscr{C}\left(\Sigma^{3}\right)$ is singular at those points $(\gamma, K)$ which generate spacetimes with spacetime Killing vector fields-i.e., those $(\gamma, K)$ such that there exists a vector field $U: \Sigma \rightarrow T \Sigma^{3}$ and a scalar field $v: \Sigma \rightarrow \mathbf{R}$ satisfying ${ }^{3}$

$$
{ }^{3} \mathscr{L}_{U} \gamma_{a b}-2 v K_{a b}=0
$$

and

$$
{ }^{3} \mathscr{L}_{U} K_{d}^{c}+v\left(R_{d}^{c}+K_{m}^{c} K_{d}^{m}\right)+\nabla^{c} \nabla_{d} v=0
$$

(see the work of Fischer, Marsden, Moncrief, and Arms $[5,6,7]) . \mathscr{A}\left(\Sigma^{3}\right)$ is singular at those points $(\lambda, \mu)$ for which there exists a "surface conformal Killing vector field" (SCKV)-i.e., there exists a vector field $V: \Sigma^{3} \rightarrow T \Sigma^{3}$ and a scalar field $\sigma: \Sigma^{3} \rightarrow \mathbf{R}^{\prime}$ such that

$$
{ }^{3} \mathscr{L}_{V} \lambda_{a b}=\sigma \lambda_{a b}
$$

and

$$
{ }^{3} \mathscr{L}_{V} \mu^{c d}=-\frac{5}{2} \sigma \mu^{c d}
$$

(see Fischer and Marsden [27]).

Are all of the singularities of $\mathscr{A}\left(\Sigma^{3}\right)$ mapped by $\mathscr{Y}_{\tau}$ to singularities of $\mathscr{C}\left(\Sigma^{3}\right)$ ? This question was raised in the context of the analysis of linearization stability of the Einstein equation (2.3). since the singularities of $\mathscr{C}\left(\Sigma^{3}\right)$ correspond to the spacetime solutions which are not linearization stable [5-7], and it was thought [8] that the singularities of $\mathscr{A}\left(\Sigma^{3}\right)$ might also have something to do with linearization instability. The answer to the question is yes, as we show in the following theorem:

Theorem 5. (Singularities of $\mathscr{A}(\Sigma)$ and the York Map). Let the vector field $V$ be a $\operatorname{SCKV}$ for the data $(\lambda, \mu) \in \mathscr{A}\left(\Sigma^{3}\right)$. Then the spacetime evolved from $\mathscr{Y}_{\tau}(\lambda, \mu)$ contains $a$ Killing field which coincides with $V$ on $\Sigma^{3}$.

2 Using the recently proven Yamabe Theorem [25] to classify Riemannian metrics as,+ 0 , or - (depending upon whether the scalar curvature may be conformally mapped to $+1,0$, or -1 ) there are solutions to (6.1) unless one of the following situations holds: a) $\tau \neq 0, \mu^{c d}=0$ everywhere on $\Sigma^{3}$ and $\lambda_{a b}$ is Yamabe class + or 0 ; b) $\tau=0, \mu^{c d}=0$ everywhere on $\Sigma^{3}$ and $\lambda_{a b}$ is Yamabe class + or - ; c) $\tau=0, \mu^{c d} \neq 0$ somewhere in $\Sigma^{3}$ and $\lambda_{a b}$ is Yamabe class 0 or -

3 The spacetime Killing vector in the evolved spacetime takes the form $U+v e_{\perp}$ at $\Sigma^{3}$ 
Proof. Equation (6.2) expresses $\left(\gamma_{a b}, K_{d}^{c}\right)=\mathscr{Y}_{\tau}\left(\lambda_{a b}, \mu^{c d}\right)$ as a functional of $\lambda_{a b}$ and $\mu^{c d}$. Thus using the chain rule together with (6.5) we obtain

$$
{ }^{3} \mathscr{L}_{v} \gamma_{a b}=v \gamma_{a b}
$$

and

$$
{ }^{3} \mathscr{L}_{V} K_{d}^{c}=-\frac{3}{2} v\left(K_{d}^{c}-\frac{1}{3} \gamma^{c} \tau\right),
$$

where $v:=\sigma+4 \nabla_{V}(\ln \varphi)$ [with $\varphi$ solving (6.1).]

The data $\left(\gamma_{a b}, K_{d}^{c}\right)$ satisfies the super Hamiltonian constraint (2.4) everywhere on $\Sigma^{3}$. Hence we may calculate

$$
\begin{aligned}
0={ }^{3} \mathscr{L}_{V}\left(R+\frac{2}{3} \tau^{2}-L^{m}{ }_{n} L^{n}\right) & =-2 \nabla^{2} v-v R-2 L^{m}{ }_{n}\left(-\frac{3}{2} v L^{n}\right) \\
& =-2 \nabla^{2} v+\left(\frac{2}{3} \tau^{2}+2 L^{m}{ }_{n} L^{n}\right) v .
\end{aligned}
$$

Multiplying (6.8) by $v$ and integrating over $\Sigma^{3}$, we get

$$
0=\int_{\Sigma^{3}}\left[(\nabla v)^{2}+\left(\frac{1}{3} \tau^{2}+L_{m}^{n} L_{n}^{m}\right) v^{2}\right],
$$

which implies that $v$ is a constant. If we now contract both sides of (6.6), we get

$$
\nabla_{a} V^{a}=\frac{3}{2} v,
$$

which, when integrated over $\Sigma^{3}$, implies that $v=0$. Thus ${ }^{3} \mathscr{L}_{V} \gamma_{a b}=0$ and from (6.7) ${ }^{3} \mathscr{L}_{V} K^{c}{ }_{d}=0$, so that $V$ generates a Killing vector field in the spacetime evolved from $\left(\gamma_{a b}, K_{d}^{c}\right)$.

Although we have only discussed the vacuum Einstein case here, the results extend to the Einstein-Yang-Mills, Einstein-Klein-Gordon, and other fields of interest.

\section{Conclusion}

Many known solutions of the Einstein equations admit proper homothetic symmetries; however, none of these are either spatially compact or asymptotically flat (except for locally flat spacetimes). Fewer solutions admit conformal symmetries which are not homothetic; a few of these, like the Friedmann-Robertson-Walker spacetimes, are spatially compact, but these all contain matter and seem very special. These are strong indications that proper conformal symmetries have little role to play in the spacetimes of general relativity, and that homothetic symmetries are useful only for model spacetimes which are neither spatially compact nor asymptotically flat. In this paper we have proved theorems supporting these assertions and we have shown by a related argument that the linearization stability criteria viewed in the space of Lichnerowicz-Choquet-Bruhat-York data agrees with the criteria in the space of Dirac-ADM data.

\section{Appendix A. Plane-Fronted Waves with Proper Conformal Killing Vectors}

In this appendix, we construct certain "plane-fronted wave" solutions of the vacuum Einstein equations with proper conformal symmetry; these constitute all solutions of these equations that admit a proper conformal symmetry. 
As discussed in the introduction, the plane-fronted wave metrics $g$ take the form (1.2a), and as long as $H$ satisfies the two dimensional Laplace equation (1.2b) then Einstein's equation is satisfied. The conformal symmetry condition requires that there exist a vector field $\xi$ and a scalar field $\sigma$ such that (4.1), or

$$
\xi^{\lambda} \partial_{\lambda} g_{\mu \nu}+\left(\partial_{\mu} \xi^{\lambda}\right) g_{\lambda \nu}+\left(\partial_{\nu} \xi^{\lambda}\right) g_{\mu \lambda}=\sigma g_{\mu \nu}
$$

is satisfied as well. We find (after a calculation) that (A.1) admits a family of solutions of the form

$$
\begin{aligned}
& \xi^{u}=u^{2} / 2-\alpha u-\beta, \\
& \xi^{r}=\delta_{A B} x^{A} x^{B} / 4-f(u)+\alpha r+\dot{c}_{A}(u) x^{A}, \\
& \xi^{A}=u x^{A} / 2+c_{A}(u)+\gamma \in_{A B} x^{B}, \quad A, B, C \ldots=(2,3) ;
\end{aligned}
$$

where $\alpha, \beta$, and $\gamma$ are arbitrary constants, and $c_{A}(u)$ and $f(u)$ are arbitrary functions of $u$; and where $H$ satisfies the equation

$$
\dot{f}-\ddot{c}_{A} x^{A}=(u-2 \alpha) H+\xi^{u} \partial_{u} H+\xi^{A} \partial_{A} H .
$$

The conformal factor $\sigma$ is equal to $u$. We further find that all solutions of (A.1) are of this form up to a constant multiplicative factor and up to a coordinate transformation.

We now show that there exist functions $H$ satisfying both (A.3) and (1.2b): Choose any constants $\alpha, \beta, \gamma$, and any smooth functions $c_{A}(u)$ and $f(u)$. Consider the linear equation (A.3) as an evolution equation for $H(u, \cdot)$ with $u$ as the time. Thus, pick any initial time $u_{0}$ such that $\xi^{u}\left(u_{0}\right) \neq 0$, and choose initial data $H\left(u_{0}, \cdot\right)$ at $u_{0}$ which satisfies (1.2b). Equation (A.3) is a linear first order equation that can be solved by the method of characteristics; as usual, we assume the coefficients and inhomogeneous terms are sufficiently smooth. A solution $H$ will then exist for any interval around $u_{0}$ during which $\xi^{u}$ does not vanish; if we choose $\beta<-\alpha^{2} / 4$ so that $\xi^{u}$ never vanishes, solutions will exist for all $u \in(-\infty, \infty)$. Taking the Laplacian $\delta^{A B} \partial_{A} \partial_{B}$ of (A.3) we get the evolution equation for the constraint (1.2b),

$$
0=\left[\xi^{u} \partial_{u}+(u-2 \alpha+1)\right]\left(\delta^{A B} \partial_{A} \partial_{B} H\right) .
$$

We thus see that (1.2b) is an involutive (or conserved) initial value constraint for (4.2), and the solution will satisfy it for all $u$ if the initial data satisfy it at $u_{0}$.

If we do not impose (1.2b), but still impose (A.2),(A.3), then we obtain some nonvacuum solutions which admit a proper conformal Killing vector. Here the stress tensor has $T_{u u}$ as its only nonvanishing component. From (A.4), $T$ satisfies the Mixed and Dominant Energy Conditions for all $u$ in the domain of definition of $H$, if it satisfies them at $u_{0}$. Such solutions are examples which are not conformally flat and not Friedmann-Robertson-Walker.

Moreover, corresponding plane-fronted wave solutions exist of the EinsteinYang-Mills equations and the massless Einstein-Klein-Gordon equations.

\section{Appendix B. The Surface Projected Lie Derivative}

Let $\Sigma_{t}$ be a family of spacelike hypersurfaces which (locally) foliate spacetime, let $B$ be a spatial tensor field defined on each surface $\Sigma_{t}$, and let $V$ be an arbitrary 
spacetime vector field defined in the region of spacetime in which the $\Sigma_{t}$ are imbedded. The surface project Lie derivative of $B$ along $V-\widetilde{\mathscr{L}}_{V} B-$ is designed to a) agree with the surface intrinsic Lie derivative of $B$ if $V$ is tangent to the hypersurface $\Sigma_{t}$, and b) describe the evolution of $B$ from surface to surface along $V$ if $V$ is transverse to the hypersurfaces. It is defined as follows: Using the appropriate spacetime covariant hypersurface projection operators [such as $P^{\mu}{ }_{v}:=g^{\mu}{ }_{v}$ $+\left(\theta^{\perp}\right)^{\mu}\left(e_{\perp}\right)_{v}$, where $\theta^{\perp}$ is the normalized one-form which annihilates vectors tangent to $\Sigma_{t}$, and $e_{\perp}$ is the hypersurface normal vector field] one extends $B$ uniquely into a spacetime covariant field $\hat{B}$. The usual spacetime covariant Lie derivative $\mathscr{L}_{V} \hat{B}$ is now well defined. Then to get $\tilde{\mathscr{L}}_{V} B$, one projects back into the hypersurface:

$$
\tilde{L}_{V} B:=\operatorname{Proj}\left(\mathscr{L}_{V} \widehat{B}\right)
$$

As an important example, consider the surface intrinsic metric $\gamma_{a b}$. One can write (omitting indices)

$$
\hat{\gamma}=g+\theta^{\perp} \otimes \theta^{\perp}
$$

Then one finds

$$
\tilde{\mathscr{L}}_{V} \gamma=i^{*} \mathscr{L}_{V}\left(g+\theta^{\perp} \otimes \theta^{\perp}\right)
$$

where the pull-back map $i^{*}$ automatically does the projection.

As another example, consider $K_{a b}$, the extrinsic curvature. One has, by defintion,

$$
\hat{K}_{a b}=\theta^{\perp}\left(\nabla_{a} \partial_{b}\right)
$$

where $\left\{\partial_{b}\right\}$ are vector fields tangent to the hypersurface $\Sigma_{t}$. Thus

$$
\tilde{\mathscr{L}}_{V} K_{a b}=i^{*} \mathscr{L}_{V} \theta^{\perp}\left(\nabla_{a} \partial_{b}\right) \text {. }
$$

Finally, note that for an object such as the curvature tensor corresponding to $\gamma_{a b}$, one may most easily calculate $\widetilde{\mathscr{L}}_{V} R_{a b}$ via the chain rule

$$
\tilde{\mathscr{L}}_{V} R_{a b}=D R_{a b}(\gamma) \cdot\left(\mathscr{L}_{V} \gamma\right) \text {. }
$$

For further discussion, see $[9,10]$.

\section{Appendix C. An Elementary Proof of Corollary 2}

Here we present an alternative, more direct proof of Corollary 2, based on some results of $\mathrm{B}$. Berger [12].

We work on the compact hypersurface $i\left(\Sigma^{3}\right)$ of constant mean curvature and use the $3+1$ split. Thus $\tau$ is constant. Berger's higher order integrability conditions [12] for the vacuum Einstein equations adjoined to the conformal Killing equations are (46), (49) and (51) of her paper:

$$
\begin{aligned}
-\nabla_{a} \nabla_{b} \sigma-K_{a b} \dot{\sigma}-\frac{1}{2} \gamma_{a b}\left(\nabla^{2} \sigma+\tau \dot{\sigma}-\ddot{\sigma}\right) & =0 \\
\nabla^{a} \nabla_{a} \sigma+\tau \dot{\sigma} & =0, \\
\nabla_{b} \dot{\sigma}+K_{a b} \nabla^{a} \sigma & =0,
\end{aligned}
$$

where $\dot{\sigma}$ and $\ddot{\sigma}$ denote respectively the first and second proper time derivatives of $\sigma$ along a timelike geodesic normal to $i\left(\Sigma^{3}\right)$ in spacetime. These are equivalent to (4.2). 
Tracing (Berger 46) and using (Berger 49) we have

$$
\begin{aligned}
\nabla_{a} \nabla_{b} \sigma+K_{a b} \dot{\sigma} & =0, \\
\ddot{\sigma} & =0 .
\end{aligned}
$$

The divergence of (Berger 51) gives

$$
\nabla^{2} \dot{\sigma}=-K_{a b} \nabla^{a} \nabla^{b} \sigma-\left(\nabla^{b} K_{a b}\right) \nabla^{a} \sigma=-K_{a b} \nabla^{a} \nabla^{b} \sigma
$$

where we have used $\operatorname{tr} K \equiv \tau=$ const and (2.5). Doubly contracting (B.1) with $K^{a b}$ and using (B.3) gives

$$
\left(-\nabla^{2}+K^{a b} K_{a b}\right) \dot{\sigma}=0,
$$

an elliptic equation for $\dot{\sigma}$. Multiplying by $\dot{\sigma}$ and integrating over $i\left(\Sigma^{3}\right)$ we have

$$
\int_{i\left(\Sigma^{3}\right)} \nabla^{a} \dot{\sigma} \nabla_{a} \dot{\sigma}+K^{a b} K_{a b} \dot{\sigma}^{2}=0
$$

since the integrand is nonnegative there are two possible cases: a) $\dot{\sigma}=0$; b) $K_{a b} \equiv 0$, $\dot{\sigma}=$ const. In case b), (2.5) is $3 \dot{\sigma}=-2 \nabla^{2} \rho$, which gives $3 \dot{\sigma} V=0$ upon integration over $i\left(\Sigma^{3}\right)$, so $\dot{\sigma}=0$ and case b) actually reduces to case a). Then from (B.1),

$$
\nabla_{a} \nabla_{b} \sigma=0 \Rightarrow \sigma=\text { const. }
$$

With $\sigma=$ const, the proof of Theorem 1 now applies and $\left(\mathscr{M}^{4}, g\right)$ is either everywhere locally flat and $g$ is of the form (1.1), or else $\xi$ is a Killing vector. In the former case, $\xi$ satisfies the integrability conditions for a spacetime homothetic Killing vector field on $i\left(\Sigma^{3}\right)$, and therefore $\xi$ is homothetic in all of spacetime (i.e., $\sigma=$ const in spacetime), by Berger's Proposition 3 [12]. In the latter case, $\xi$ satisfies the integrability conditions for a spacetime Killing vector on $i\left(\Sigma^{3}\right)$, and similarly must be Killing $(\sigma=0)$ throughout spacetime, by a result of Moncrief [28].

\section{Appendix D. A Friedmann-Robertson-Walker-Yang-Mills Solution}

Here we will show that the Einstein-Yang-Mills equations admit $k=+1$ Friedmann-Robertson-Walker solutions, at least if the gauge group contains a subgroup $S U(2)$ or $U(1) \otimes U(1) \otimes U(1)$. These can be constructed as follows for $\mathrm{SU}(2)$. Let $\sigma_{I}(I, J, K, \ldots=1,2,3)$ be a basis of the subalgebra su(2), with commutation relations $\left[\sigma_{I}, \sigma_{J}\right]=\varepsilon_{I J K} \sigma_{K}$. The $k=+1$ Friedmann-Robertson-Walker metric is

$$
d s^{2}=-d t^{2}+a^{2}(t) d \Sigma^{3}
$$

where $d \Sigma^{2}$ is the metric of the unit 3-sphere. On the unit 3-sphere there exist three 1forms $\omega^{I}$ that satisfy

$$
d \omega^{I}=\varepsilon_{I J K} \omega^{J} \wedge \omega^{K}
$$

such that

$$
d \Sigma^{2}=\delta_{I J} \omega^{I} \otimes \omega^{J}
$$

(see, e.g. [13], §30.7). Let the gauge potential 1-form $\mathbf{A}$ for spacetime be of the form

$$
\mathbf{A} \equiv A_{\mu} d x^{\mu}=\alpha(t) \sigma_{I} \boldsymbol{\omega}^{I},
$$


where $\alpha$ is a function of time alone. Then a straightforward calculation shows that solutions to the Einstein equations coupled to the Yang-Mills equations (3.13) exist of the form (D.1), (D.2) as long as $\alpha$ and $a$ satisfy certain coupled ordinary differential equations in $t$. Similar examples exist for a subalgebra $u(1) \otimes u(1)$, with three $\sigma_{I}$ being elements from the three $u(1)$. These examples are amusing in that the gauge field $F$ does not share the full $\mathrm{SO}(4)$ spatial isometry of spacetime; rather $F$ is invariant under only one of the $\mathrm{SO}(3)$ factors in the direct product $\mathrm{SO}(4)=$ $\mathrm{SO}(3) \otimes \mathrm{SO}(3)$. The stress tensor $T$ is invariant under $\mathrm{SO}(4)$, of course.

(More generally, one gets Bianchi IX ("Mixmaster") solutions ([13], §30.7) upon replacing (D.2) by $\mathbf{A}=\Sigma_{I} \alpha_{I}(t) \sigma_{I} \boldsymbol{\omega}^{I}$, where the $\alpha_{I}(t)$ are three different functions of time alone. Then, $g, T$ and $F$ all have the same isometry group $\operatorname{SO}(3)$. These solutions do not in general admit any proper conformal Killing vector fields.)

Acknowledgements. This work was supported in part by National Science Foundation under Grant Nos. DMS 83-03998 at the University of Oregon, DMS 84-04506 at the University of California at Berkeley, PHY 85-03072 at Yale, and PHY 85-06686 and PHY 82-17853 (supplemented by founds from the National Aeronautics and Space Administration) at the University of California at Santa Barbara.

\section{References}

1. Gerhardt, C.: H-surfaces in Lorentcian manifolds. Commun. Math. Phys. 89, 523 (1983)

2. Marsden, J., Tipler, F.: Maximal hypersurfaces and foliations of constant mean curvature in general relativity. Phys. Rep. 66, 109 (1980)

3. Löbell, F.: Ber. Verhandl. Sächs Akad. Wiss. Leipzig. Math. Phys. K1. 83, 167 (1931)

4. Ehlers, J., Kundt, W.: Exact solutions of the gravitational field equations. In: Gravitation: An introduction to current research. Witten, L. (ed.). New York: Wiley 1962

5. Fisher, A., Marsden, J.: Bull. Am. Math. Soc. 79, 995 (1973)

6. Fischer, A., Marsden, J., Moncrief, V.: The structure of the space of solutions of Einstein's equations. I. One Killing field. Ann. Inst. H. Poincaré 33, 147 (1980)

7. Arms, J., Marsden, J., Moncrief, V.: The structure of the space of solutions of Einstein's equations. II. Several Killing fields and the Einstein-Yang-Mills equations. Ann. Phys. (N.Y.) 144, 81 (1982)

8. O'Murchadha, N., York, J. W.: Initial-value problem of general relativity. II. Stability of solutions of the initial-value equations. Phys. Rev. D 10, 437 (1974)

9. Isenberg, J., Nester, J.: Canonical gravity. In: General relativity and gravitation. Vol. 1. Held, A. (ed.). New York, London: Plenum 1980

10. Fischer, A., Marsden, J.: The initial value problem and the dynamical formulation of general relativity. In: General relativity. Einstein centenary survey. Hawking, S., Israel, W. (eds.). Cambridge: Cambridge University Press 1979

11. Choquet-Bruhat, Y., York, J.: The Cauchy problem. In: General relativity and gravitation. Vol. 1. Held, A. (ed.). New York, London: Plenum 1980

12. Berger, B. K.: Homothetic and conformal motions in spacelike slices of solutions of Einstein's equations. J. Math. Phys. 17, 1268 (1976)

13. Misner, C., Thorne, K. Wheeler, J.: Gravitation. San Francisco: W. H. Freemann 1973

14. Hawking, S. W., Ellis, G. F. R.: The large scale structure of spacetime. Cambridge: Cambridge University Press 1983

15. Eardley, D. M.: Self-similar spacetimes: Geometry and dynamics. Commun. Math. Phys. 37, 287 (1974)

16. Yano, K., Bochner, S.: Curvature and Betti numbers. In: Ann. Math. Stud. No. 32. Princeton: Princeton University Press 1953

17. DeWitt, B.: In: Relativity, group and topology. DeWitt, C. DeWitt, B. (eds.) Gordon and Breach 1964

18. Yano, K.: The theory of Lie derivatives and its applications. New York: Interscience 1957 
19. Garfinkle, D., Tiem, Q. J.: private communication 1985

20. Schoen, R., Yau, S. -T.: Proof of the positive mass theorem. II. Commun. Math. Phys. 79, 231 (1981), and their previous papers cited therein

21. Witten, E.: A new proof of the positive energy theorem. Commun. Math. Phys. 80, 381 (1981)

22. Parker, T., Taubes, C. H.: On Witten's proof of the positive energy theorem. Commun. Math. Phys. 84, 223 (1982)

23. Yip, P.: private communication 1985

24. Maithreyan, T. Eardley, D.: Gravitational collapse of spherically symmetric scale invariant scalar fields. ITP preprint 1985

25. Schoen, R.: Conformal deformation of a Riemannian metric to constant scalar curvature. J. Differen. Geom. (To appear).

26. Isenberg, J., Marsden, J.: Geom. Phys. 1, 85 (1984)

27. Fischer, A., Marsden, J.: Can. J. Math. 29, 193 (1977)

28. Moncrief, V.: Spacetime symmetries and linearization stability of the Einstein equations. I. J. Math. Phys. 16, 493 (1975)

29. Jantzen, R., Rosquist, K.: Adapted Slicings of space-times possessing simply transitive similarity groups. J. Math. Phys. 27, 1191 (1986).

30. Hsu, L., Wainwright, J.: Self-similar spatially homogeneous cosmologies I: Orthogonal perfect fluid and vacuum solutions. Class. Quantum Gravity. (To appear).

31. Garfinkle, D.: Asymptotically flat spacetimes have no conformal Killing fields. J. Math. Phys. (To appear).

Communicated by S. -T. Yau

Received December 16, 1985

Note added in proof. For recent work on solutions admitting a proper homothetic Killing vector field see $[29,30]$. For a result on nonexistence of spacetimes that are asymptotically flat at null infinity and that admit a conformal Killing vector field see [31]. 